QUARTERLY OF APPLIED MATHEMATICS

VOLUME LXIII, NUMBER 3

SEPTEMBER 2005, PAGES 469-478

S 0033-569X(05)00966-1

Article electronically published on April 11, 2005

\title{
ON THE DERIVATION OF BOUNDARY CONDITIONS FROM THE GLOBAL PRINCIPLES OF CONTINUUM MECHANICS
}

\author{
BY \\ GERALD G. KLEINSTEIN \\ 10 Emerson Place, Boston MA 02114
}

\begin{abstract}
We consider the motion of a fluid exterior to a moving rigid obstacle, or interior to a moving rigid shell. The boundary conditions, such as the no-slip condition and the condition of an isothermal wall, applied in the solution of the system of differential equations describing these motions, are currently assumed to be an approximation derived from experimental observation rather than an exact law. It is the purpose of this paper to show that the boundary conditions at a material interface between a fluid and a solid are derivable from the global principles of balance of continuum mechanics and the Clausius-Duhem inequality.
\end{abstract}

1. Introduction. It is generally accepted that the boundary conditions prescribed in the solution of the differential equations of fluid mechanics, such as the no-slip condition and the isothermal wall condition, are derived from experimental observation. According to Lamb (5] p. 576), "It appears probable that in all ordinary cases there is no motion, relative to the solid, of the fluid immediately in contact with it." In his discussion of "proper boundary conditions", Truesdell (9] pp. 138 and 147) points out the frail mathematical status of the no-slip condition. A similar attitude is also expressed by Batchelor ([1, p. 149): "the condition of continuity of the velocity is thus not an exact law, but a statement of what may be expected to happen, approximately, in normal circumstances."

It is obviously desirable, in a theory of rational fluid mechanics, that such information as the boundary conditions should be deduced based on theoretical grounds. We propose here that all the information required for the boundary conditions is contained within the formulation of the global principles of continuum mechanics, which hold in the presence of interior surfaces of discontinuity in the domain, such as material interfaces or shock waves.

Received June 30, 2004.

2000 Mathematics Subject Classification. Primary 76A02; Secondary 74A15.

Key words and phrases. Navier-Stokes equations, boundary conditions, entropy condition.

E-mail address: ggkleinstein@rcn.com

(C)2005 Brown University 
As shown by Kleinstein [4, by applying Kotchine's theorem (see Truesdell \& Toupin [8], p. 527), the global system consisting of integral equations can be expressed in terms of an equivalent system of differential equations and jump conditions.

Analyzing the jump conditions at a material interface between a solid and a viscous heat-conducting fluid, we show that if the solid is not thermally insulated and the component of the viscous stress vector in the tangent plane is not zero, the vanishing of the entropy production per unit area at the interface constitutes a necessary and sufficient condition for an isothermal interface and for the no-slip condition. A boundary condition, which constitutes a necessary condition for slip, is also derived.

2. Definitions and Hypotheses. We make the following hypotheses:

H1. The domain of flow includes the fluid and an infinitesimal layer of the solid surface.

H2. The global principles of continuum mechanics (conservation of mass, momentum balance, energy balance, and the Clausius-Duhem inequality), hold in the entire flow domain.

H3. The velocity vector, temperature, heat transfer vector, and the stress vector at the surface of the solid are well defined.

H4. The standard constitutive equations of viscous heat-conducting fluid dynamics (see Section (5)) are satisfied.

H5. The solid is a rigid body with a piecewise smooth surface.

H6. The temperature $T$ and the density $\rho$ are positive.

We also make the following definitions:

D1. An isothermal interface is a material interface with a continuous temperature across it.

D2. An isentropic interface is a material interface with a zero rate of entropy production per unit area.

3. The global system. Let the variables $\rho, u_{i}, t_{i j}, e, q_{i}, s, T$, and $g_{i}$ designate the density, velocity vector, stress tensor, internal energy, heat transfer vector, entropy, temperature, and specific body force, respectively. The principles of balance of continuum mechanics, denoting conservation of mass and balance of momentum and energy, are 
given by the integral equations:

$$
\begin{gathered}
\frac{D}{D t} \int_{V} \rho d v=0 \\
\frac{D}{D t} \int_{V} \rho u_{i} d v=\int_{\partial V} t_{i j} n_{j} d a+\int_{V} \rho g_{i} d v \\
\frac{D}{D t} \int_{V} \rho\left(e+\frac{1}{2} u_{i} u_{i}\right) d v=-\int_{\partial V} q_{i} n_{i} d a+\int_{\partial V} t_{i j} u_{i} n_{j} d a+\int_{V} \rho g_{i} u_{i} d v .
\end{gathered}
$$

The Clausius-Duhem inequality is also assumed in the form

$$
\frac{D}{D t} \int_{V} \rho s d v+\int_{\partial V}\left(q_{i} n_{i} / T\right) d a=\Gamma \geq 0
$$

In the above equations, $D / D t$ denotes the material derivative, $n_{i}$, the exterior normal to the surface enclosing an arbitrary volume $V$ of the fluid, and $\Gamma$ is the rate of entropy production in the volume. An exposition of the global principles of continuum mechanics can be found, for example, in Serrin [7, Truesdell ([9], [10]), and Gurtin 2].

4. An equivalent differential system. Under hypothesis $\mathrm{H} 3$, the global equations (3.1) to (3.4) can be expressed in terms of an equivalent system of differential equations and jump conditions (see Truesdell [10, p. 164). By definition, the jump conditions relate properties at points on opposite sides of a singular surface. We denote such jumps with the symbol [ ]. If $s$ and $f$ correspond to the solid and fluid sides of the interface respectively, then we define

$$
\left[b_{i}\right] n_{i}^{s}=\left(b_{i}^{f}-b_{i}^{s}\right) n_{i}^{s},
$$

where $n_{i}^{s}$ is a unit vector oriented from the solid to the fluid side of the interface.

A derivation of the jump conditions for a material interface, for a change of phase, and for shock waves was given by Keller [3]; a more extensive exposition of the subject of singular surfaces is to be found in Truesdell \& Toupin [8]. Indeed by Kotchine's theorem (see Truesdell \& Toupin [8, p. 527), the global equations are equivalent to a system consisting of a set of differential equations and a set (or sets) of jump conditions. The differential equations are:

$$
\begin{gathered}
\frac{D \rho}{D t}=-\rho \frac{\partial u_{i}}{\partial x_{i}}, \\
\rho \frac{D u_{i}}{D t}=\frac{\partial t_{i j}}{\partial x_{j}}+\rho g_{i}, \\
\rho \frac{D e}{D t}=-\frac{\partial q_{i}}{\partial x_{i}}+t_{i j} \frac{\partial u_{i}}{\partial x_{j}}, \\
\rho \frac{D s}{D t}+\frac{\partial\left(q_{i} / T\right)}{\partial x_{i}}=\gamma_{v} \geq 0,
\end{gathered}
$$


where in equation (4.4), $\gamma_{v}$ is the rate of entropy production per unit volume. For a fluid-solid interface, the jump conditions are

$$
\begin{gathered}
{\left[u_{i}\right] n_{i}^{s}=0,} \\
{\left[t_{i j}\right] n_{i}^{s}=0,} \\
{\left[-t_{i j} u_{i}+q_{j}\right] n_{j}^{s}=0,}
\end{gathered}
$$

and

$$
\left[q_{i} / T\right] n_{i}^{s}=\gamma_{s} \geq 0
$$

The jump condition (4.5) expresses the continuity of the normal velocity component and asserts that the fluid does not penetrate the solid. Equation (4.6) is the Poisson condition (see Truesdell, [10]) for the continuity of the stress vector, and equation (4.7) expresses the balance of energy across the interface. Equation (4.8) is the ClausiusDuhem jump condition - a statement of the second law for the interface. The rate of entropy production per unit area, $\gamma_{s}$, in (4.8) is positive when processes within the interface are irreversible and it is zero when reversible. We will call a material interface with a zero entropy production per unit area an isentropic interface (D2). Along an isentropic interface the entropy may vary.

A motion satisfying (4.1), (4.2), (4.3) and (4.5), (4.6), (4.7) will be called mechanicallyadmissible. A mechanically-admissible motion that satisfies the Clausius-Duhem inequality (3.4) is called thermodynamically admissible, and a mechanically-admissible motion that is incompatible with the Clausius-Duhem inequality will be called thermodynamically inadmissible. A thermodynamic inadmissibility constitutes a sufficient condition for the failure of a mechanically-admissible motion to persist in time.

5. The constitutive equations. In order to complete our definitions, we introduce the standard constitutive equations of a viscous heat-conducting fluid (see Serrin [7] and Gurtin [2]). The requisite constitutive equations are the following:

$$
\begin{gathered}
e=e(\rho, T), \\
s=s(\rho, T), \\
t_{i j}=-p \delta_{i j}+\tau_{i j},
\end{gathered}
$$

where

$$
\tau_{i j}=2 \mu e_{i j}+\lambda \Theta \delta_{i j}
$$

and

and where

$$
q_{i}=-k \frac{\partial T}{\partial x_{i}}
$$

$$
e_{i j}=\frac{1}{2}\left(\frac{\partial u_{i}}{\partial x_{j}}+\frac{\partial u_{j}}{\partial x_{i}}\right) \text { and } \Theta=\frac{\partial u_{i}}{\partial x_{i}} .
$$

In the above equations, the variables $p, \tau_{i j}, e_{i j}, \mu, \mu_{v}$, and $k$ denote pressure, viscous stress tensor, rate of deformation tensor, viscosity coefficient, the coefficient of bulk viscosity $\mu_{v}=\lambda+2 \mu / 3$, and the heat transfer coefficient, respectively. The symbol $\delta_{i j}$ is the Kronecker delta, and $\Theta$ is the divergence of the velocity field. The temperature $T$ 
and the density $\rho$ are assumed positive (H6). We suppose as well that $p, \mu, \lambda$, and $k$ depend on $(\rho, T)$ and that the Gibbs relation,

$$
T d s=d e+p d\left(\frac{1}{\rho}\right)
$$

holds; for internal points in the domain, this relation can also be written as

$$
T \frac{D s}{D t}=\frac{D e}{D t}+p \frac{D}{D t}\left(\frac{1}{\rho}\right) .
$$

Substituting the continuity equation (4.1), the energy equation (4.3), and the definition of the viscous stress tensor (5.3) into equation (5.7) gives

$$
\rho \frac{D s}{D t}+\frac{\partial\left(q_{i} / T\right)}{\partial x_{i}}=\frac{1}{T}\left(\tau_{i j} \frac{\partial u_{j}}{\partial x_{i}}-\frac{q_{i}}{T} \frac{\partial T}{\partial x_{i}}\right) .
$$

Comparing equation (5.8) with (4.4) identifies the entropy production per unit volume, $\gamma_{v}$, as

$$
\gamma_{v}=\frac{1}{T}\left(\tau_{i j} \frac{\partial u_{j}}{\partial x_{i}}-\frac{q_{i}}{T} \frac{\partial T}{\partial x_{i}}\right) \geq 0 .
$$

Equation (5.9) is a constraint that must be satisfied when selecting the constitutive equations. It shows, furthermore, that under normal circumstances at local thermodynamic equilibrium, entropy production per unit volume is positive at interior points of the fluid. As shown by Serrin [7, pp. 237, 238 and Truesdell [9], p. 46, if the two viscosity coefficients $\mu_{v}$ and $\mu$, and the heat transfer coefficient $k$ satisfy the inequalities

$$
0 \leq \mu_{v}=\lambda+2 \mu / 3<\infty, \quad 0 \leq \mu<\infty \text { and } 0 \leq k<\infty,
$$

equation (5.9), and consequently the differential Clausius-Duhem inequality (4.4), are satisfied identically.

The inequalities in (5.10) guarantee only the differential form of the Clausius-Duhem inequality, not the global form. Thus, for a solution of the differential system to be compatible with the global balance equations and the global Clausius-Duhem inequality (3.4), the boundary conditions must still comply with all four jump conditions (4.5) to (4.8). As shown below, it is the compatibility requirement with the Clausius-Duhem inequality (4.8) that determines the required boundary conditions.

6. Differential equations, jump conditions, and boundary conditions. Substituting the constitutive equations (5.1) to (5.5) into equations (4.1) to (4.3) results in the familiar set of the Navier-Stokes equations of motion. Substituting (5.4) and (5.5) into (5.9) gives the entropy production per unit volume as

$$
\gamma_{v}=\frac{1}{T}\left(2 \mu\left(e_{i j}-\frac{1}{3} \delta_{i j} \Theta\right)^{2}+\mu_{v} \Theta^{2}+\frac{k}{T} \frac{\partial T}{\partial x_{i}} \frac{\partial T}{\partial x_{i}}\right) .
$$

We turn now to the derivation of the boundary conditions from the jump conditions. Substituting the constitutive equations into the jump conditions leaves equation (4.5) unaltered. 
Utilizing (5.3) and (5.4) in jump condition (4.6) determines the stress vector on the solid surface in terms of the pressure and the viscous stress vector of the fluid at the interface as

$$
t_{i j}^{s} n_{i}^{s}=-p n_{j}^{s}+\tau_{i j} n_{j}^{s}=-p n_{j}^{s}+\left(2 \mu e_{i j}+\lambda \Theta \delta_{i j}\right)^{f} n_{i}^{s} .
$$

By the continuity of the stress vector, equation (4.6), and the definition of the viscous stress tensor, equation (5.3), the energy jump condition (4.7) becomes

$$
\left[q_{i}\right] n_{i}^{s}=t_{i j} n_{i}^{s}\left[u_{j}\right]=\left\{(-p+\lambda \Theta) \delta_{i j}+2 \mu e_{i j}\right\} n_{i}^{s}\left[u_{j}\right]
$$

and by use of equation (4.5),

$$
\left[q_{i}\right] n_{i}^{s}=\tau_{i j} n_{i}^{s}\left[u_{j}\right]=2 \mu e_{i j} n_{i}^{s}\left[u_{j}\right] .
$$

Now writing out jump condition (4.8) in the form

$$
\left[q_{i} / T\right] n_{i}^{s}=\frac{1}{T^{f}}\left[q_{i}\right] n_{i}^{s}+q_{i}^{s} n_{i}^{s}\left[\frac{1}{T}\right] \geq 0,
$$

then substituting from equation (6.3), we obtain the Clausius-Duhem jump condition as

$$
\gamma_{s}=\frac{1}{T^{f}} \tau_{i j} n_{i}^{s}\left[u_{j}\right]+q_{i}^{s} n_{i}^{s}\left[\frac{1}{T}\right]=\frac{2 \mu}{T^{f}} e_{i j} n_{i}^{s}\left[u_{j}\right]+q_{i}^{s} n_{i}^{s}\left[\frac{1}{T}\right] \geq 0 .
$$

This result, expressing the Clausius-Duhem condition in terms of both thermal and mechanical variables, is a central point for this analysis.

A solution of the Navier-Stokes equations, $\left(u_{i}, \rho, T\right)$, which at the material interface satisfies the boundary condition (4.5), can always comply with equations (6.2) and (6.3) and as such it describes a mechanically-admissible motion. If such a solution also satisfies the no-slip boundary condition, $u_{i}^{f}=u_{i}^{s}$, and the isothermal interface condition, $T^{f}=$ $T^{s}$, the Clausius-Duhem jump condition (6.4) is satisfied identically and thus the solution is thermodynamically admissible with an isentropic interface.

It follows then that the no-slip condition and the isothermal interface condition constitute sufficient conditions for a solution of the Navier-Stokes equation to be thermodynamically admissible with an isentropic interface. It may be noted that the no-slip condition, $u_{i}^{f}=u_{i}^{s}$, implies the continuity of the normal velocity component, which was already established by equation (4.5). Thus the contribution of the Clausius-Duhem jump condition is to assert the continuity of the tangential velocity component as well as the continuity of the temperature.

Clearly, equation (6.4) admits other boundary conditions. For example, when irreversible processes arise at the interface due to non-equilibrium states, discontinuous values of the temperature and velocity are admissible, which in turn contribute to the entropy production, $\gamma_{s}$. While the rate of entropy production per unit area for such processes can be computed by (6.4), the actual value of the jump in either the temperature or the velocity cannot be determined by continuum mechanics and must be supplemented by microscopic theories. The earliest contributions made to evaluate these discontinuities are attributed to Maxwell [6], who used kinetic theory. Restricting the analysis to the case of local equilibrium states everywhere in the domain (H1), it is assumed that there is a zero rate of entropy production at the interface and as defined in D2 it is called an isentropic interface. 
In what follows, we propose to establish the required constraints for an isentropic interface to be a necessary and sufficient condition for an isothermal interface and for a no-slip condition. In order to arrive at this result, we first consider the following Lemma.

Lemma. The Clausius-Duhem jump condition (6.4), which may be written as

$$
\gamma_{s}=\gamma_{s}^{T}+\gamma_{s}^{W} \geq 0
$$

where

$$
\gamma_{s}^{T}=q_{i}^{s} n_{i}^{s}\left[\frac{1}{T}\right]
$$

is the rate of entropy production per unit area associated with heat transfer processes at the interface and

$$
\gamma_{s}^{W}=\frac{1}{T^{f}} \tau_{i j} n_{i}^{s}\left[u_{j}\right]
$$

is the rate of entropy production per unit area associated with viscous work rate at the interface; is equivalent to the two inequalities

$$
\gamma_{s}^{T}=q_{i}^{s} n_{i}^{s}\left[\frac{1}{T}\right] \geq 0
$$

and

$$
\gamma_{s}^{W}=\frac{1}{T^{f}} \tau_{i j} n_{i}^{s}\left[u_{j}\right] \geq 0
$$

Proof. Suppose $\gamma_{s}^{T}$ is negative; then

$$
\gamma_{s}^{T}=q_{i}^{s} n_{i}^{s}\left[\frac{1}{T}\right]=q_{i}^{s} n_{i}^{s}\left(\frac{T^{s}-T^{f}}{T^{s} T^{f}}\right)<0,
$$

which states that if $q_{i}^{s} n_{i}^{s}$, the rate of heat transfer from the solid to the interface, is positive, then $T^{s}-T^{f}$ is negative, which contradicts Clausius' statement of the second law; hence $\gamma_{s}^{T} \geq 0$.

Suppose $\gamma_{s}^{W}$ is negative; then by equation (6.3), we can write

$$
\gamma_{s}^{W}=\frac{1}{T^{f}} \tau_{i j} n_{i}^{s}\left[u_{j}\right]=\frac{1}{T^{f}}\left[q_{i}\right] n_{i}^{s}<0 .
$$

Now the term $\tau_{i j} n_{i}^{s}\left[u_{j}\right]$ represents the net rate of viscous work done by the fluid on the interface, while $\left[q_{i}\right] n_{i}^{s}$ is the net rate of heat transferred out of the interface. The inequality above implies that the net positive rate of heat transferred into the interface is converted directly into the rate of viscous work done by the interface on the fluid, which contradicts Kelvin's statement of the second law; hence $\gamma_{s}^{W} \geq 0$. This completes the proof.

Corollary. A necessary and sufficient condition for both $\gamma_{s}^{T}$ and $\gamma_{s}^{W}$ to be zero is that the entropy production per unit area at the interface, $\gamma_{s}$, is equal to zero.

Proof. Let

$$
\gamma_{s}=\gamma_{s}^{T}+\gamma_{s}^{W}=0
$$


If $\gamma_{s}^{T}$ and $\gamma_{s}^{W}$ are both zero, then clearly $\gamma_{s}$ is zero. Suppose $\gamma_{s}$ is zero; then if $\gamma_{s}^{T}$ is positive, by (6.7) $\gamma_{s}^{W}$ must be negative, which violates (6.6) as shown in the Lemma. Consequently

$$
\gamma_{s}^{T}=q_{i}^{s} n_{i}^{s}\left[\frac{1}{T}\right]=0
$$

and again by (6.7)

$$
\gamma_{s}^{W}=\frac{1}{T^{f}} \tau_{i j} n_{i}^{s}\left[u_{j}\right]=\frac{2 \mu}{T^{f}} e_{i j} n_{i}^{s}\left[u_{j}\right]=0,
$$

which completes the proof.

While equation (6.8) is set in a convenient form for the discussion of the continuity of temperature at the interface, equation (6.9) requires some significant simplification before the conditions for no-slip can be considered. We propose to express the scalar product between the vectors $e_{i j} n_{i}^{s}$ and $\left[u_{j}\right]$ in terms of the relative velocity

$$
v_{j}=u_{j}-u_{j}^{s}=q^{r} t_{j}^{r},
$$

its magnitude $q^{r}=\left[\left(u_{j}-u_{j}^{s}\right)\left(u_{j}-u_{j}^{s}\right)\right]^{1 / 2}$, and in terms of some additional properties associated with the geometry of the solid surface $S$.

Let $S$ be the fluid-solid interface of a rigid body moving in a fluid of infinite extent or a fluid-solid interface of a rigid shell enclosing a fluid. Let the velocity of the rigid body be defined by

$$
u_{j}^{s}=w_{j}+\epsilon_{j k l} \Omega_{k}\left(x_{l}-x_{l 0}\right),
$$

where $w_{j}$ is the translational velocity, $x_{l 0}$ the coordinate of the instantaneous center of rotation, $x_{l}$ the coordinate of a point on the surface $S$, and $\Omega_{k}$ the instantaneous angular velocity. Since the rate-of-deformation tensor $e_{i j}$ is invariant under the velocity transformation (6.10), we obtain

$$
e_{i j}=e_{i j}^{r}=\frac{1}{2}\left(\frac{\partial v_{i}}{\partial x_{j}}+\frac{\partial v_{j}}{\partial x_{i}}\right),
$$

and thus at the interface where $v_{j}=u_{j}^{f}-u_{j}^{s}=\left[u_{j}\right]$, we have

$$
e_{i j}\left[u_{i}\right]=e_{i j}^{r} v_{i} .
$$

Let $C$ be a streamline for the relative velocity $v_{j}$, defined by the integral curve

$$
\frac{d x_{j}}{d \xi}=v_{j}\left(x_{1}, x_{2}, x_{3}, t\right),
$$

where $\xi$ is a parameter along this streamline which passes through the point $P_{0}$ of the interface. Following Kleinstein [4], p. 104, by using $v_{j}=q^{r} t_{j}^{r}$, we obtain

$$
2 e_{i j}^{r} n_{i}^{s} t_{j}^{r}=\left(\frac{\partial q^{r}}{\partial n}+\kappa_{n} q^{r}\right),
$$

where $\partial / \partial n$ designates the normal derivative interior to the fluid, and $\kappa_{n}$ is the normal curvature of the surface curve $C$. In particular, if $u$ and $v$ are two principal directions at the point $P_{0}$ of the interface, by Euler's theorem (Weatherburn [11]),

$$
\kappa_{n}=\kappa_{u} \cos ^{2} \psi+k_{v} \sin ^{2} \psi,
$$


where $k_{u}$ and $k_{v}$ are the corresponding two principal curvatures at $P_{0}$, and $\psi$ is the angle that the streamline of the relative velocity makes with the principal direction $d v=0$.

Utilizing equation (6.13), equation (6.6) can be finally written as

$$
\gamma_{s}^{W}=\frac{q^{r}}{T^{f}} \tau^{r} \geq 0
$$

where the scalar $\tau^{r}$, the component of the viscous stress vector in the direction of the relative velocity, is now given by

$$
\tau^{r}=\tau_{i j} n_{i}^{s} t_{j}^{r}=\mu\left(\frac{\partial q^{r}}{\partial n}+\kappa_{n} q^{r}\right)^{f} .
$$

Consideration of equations (6.14) and (6.15) shows that when $q^{r} \neq 0$, thermodynamic admissibility requires $\tau^{r} \geq 0$. We are now well posed to formulate the following theorem.

No-SliP TheOrem. Let $S$ be a fluid-solid interface of a rigid body moving in a fluid of infinite extent, or a fluid-solid interface of a rigid shell enclosing a fluid and let the velocity of $S$ be prescribed by the rigid body motion (6.11). Then, if the solid is not thermally insulated and $\tau^{r}$ is not zero, an isentropic interface constitutes a necessary and sufficient condition for the continuity of velocity and temperature at the interface.

Proof. Suppose the no-slip condition $\left[u_{i}\right]=0$ holds and the isothermal interface condition $[T]=0$ applies. Then, since if $\left[u_{i}\right]=0, q^{r}$ is also zero, we obtain

$$
\gamma_{s}=\frac{q^{r}}{T^{f}} \tau^{r}+q_{i}^{s} n_{i}^{s}\left[\frac{1}{T}\right]=0
$$

which proves the sufficiency condition.

Now let $\gamma_{s}$ be zero. Then by the Corollary, equation (6.8), $\gamma_{s}^{T}$ is also zero. Assuming the wall is not insulated, i.e., $q_{j}^{s} n_{j}^{s}$ is non-zero, then, again by equation (6.8), the temperature at the interface must be continuous.

Let $\gamma_{s}$ be zero. Then by the Corollary, equation (6.9), $\gamma_{s}^{W}$ is also zero. Since by assumption $\tau^{r}$ is not zero, then by (6.14) $q^{r}$ is zero, and hence the velocity is continuous at the solid fluid interface. This completes the proof.

Note. As can be seen from the jump condition (6.3), the no-slip condition implies the continuity of the normal component of the heat transfer vector across the interface.

It furthermore follows from equation (6.14) that the condition $\tau^{r}=0$ constitutes a necessary condition for slip. Thus, in a mechanically-admissible motion for an inviscid fluid where $\mu=0$, see equation (5.10), or in a mechanically-admissible motion for a viscous heat-conducting fluid which satisfies the boundary condition

$$
\left(\frac{\partial q^{r}}{\partial n}+\kappa_{n} q^{r}\right)^{f}=0
$$

at the interface, slip is admissible and the resulting motion is thermodynamically admissible. 
In sum, by utilizing the global principles of continuum mechanics, the constraints under which the no-slip (or slip) condition and an isothermal interface hold at a fluidsolid interface have been determined for a fluid, subject to the standard constitutive equations of a viscous heat-conducting fluid dynamics.

Acknowledgment The author wishes to thank Prof. Lu Ting of the Courant Institute of Mathematical Sciences for his valuable discussions.

\section{REFERENCES}

[1] Batchelor, G. K., An Introduction to Fluid Dynamics, Cambridge University Press, 1967. MR 1744638 (2000j:76001)

[2] Gurtin, M. E., An Introduction to Continuum Mechanics, Academic Press, 1981. MR0636255 (84c:73001)

[3] Keller, J. B., Geometrical acoustics I. The theory of weak shock waves, J. Appl. Physics, 1954, 25, 938-947. MR0067654 (16,761e)

[4] Kleinstein, G. G., On the non-persistence of irrotational motion in a viscous heat-conducting fluid, Arch. Ratl. Mech. Anal., 1988, 101 (No. 2), 95-105. MR0921933 (89c:76024)

[5] Lamb, H., Hydrodynamics, Cambridge University Press, 1932. MR.1317348 (96f:76001)

[6] Maxwell, C., Scientific Papers of Clark Maxwell, vol 2, p.1, Dover, New York, 1860.

[7] Serrin, J., Mathematical Principles of Classical Fluid Mechanics, sections 58-65. Handbuch der Physik VIII/1, Springer-Verlag, 1959. MR0108116 (21:6836b)

[8] Truesdell, C., \& R. Toupin, The Classical Field Theories, pp. 492-530. Handbuch der Physik III/1, Springer-Verlag, 1960. MR0118005 (22:8778)

[9] Truesdell, C., The Mechanical Foundations of Elasticity and Fluid Dynamics, Gordon and Breach, 1966. MR0192962 (33:1187a)

[10] Truesdell, C., The Elements of Continuum Mechanics, Springer-Verlag, 1966. MR0214315(35:5166)

[11] Weatherburn, C. E., Differential Geometry of Three Dimensions, Vol. 1, Cambridge University Press, 1955. 